\title{
Anatomical variations of the palmaris longus muscle including its relation to the median nerve - a proposal for a new classification
}

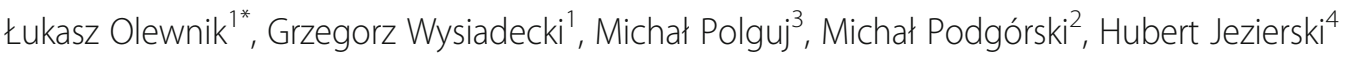 \\ and Mirosław Topol ${ }^{1}$
}

\begin{abstract}
Background: The palmaris longus (PL) muscle is characterised by high morphological diversity, and its tendon crosses the median nerve (MN) at different levels. Due to the fact that the palmaris longus tendon is routinely harvested for reconstruction of other tendons, knowledge of its morphological variations is clinically important. Therefore, the purpose of the study was to suggest a new morphological classification of the PL muscle and characterise the relationship of its tendon to the median nerve.

Methods: Standard dissection was performed on 80 randomised and isolated upper limbs (40 left and 40 right) fixed in a $10 \%$ formalin solution. Measurements of muscle belly and tendon were obtained. The course and location of tendon insertion, as well as its relationship to the median nerve, were noted.

Results: The palmaris longus muscle was present in $92.5 \%$ of specimens. Three types of palmaris longus muscle were identified based on the morphology of its insertion (types I-III) and these were further subdivided into three subgroups ( $A-C$ ) according to the ratio of the length of the muscle belly and its tendon. The most frequent was type I (78.8\%), where the tendon attached to the palmar aponeurosis, and subtype B, where the tendon-to-belly ratio was 1-1.5 (41.1\%). The mean distance from the interstyloid line to the crossing between the median nerve and the palmaris longus tendon was $31.6 \mathrm{~mm}$. In addition, two types of palmaris longus were described.
\end{abstract}

Conclusion: The presented classification of palmaris longus muscle types allows a better characterization of its diversity and may be useful in planning tendon grafting.

Keywords: Palmaris longus muscle, Palmaris longus tendon, Tendon grafts, New classification

\section{Background}

The palmaris longus (PL) is a narrow, fusiform muscle of the superficial anterior compartment of the forearm [1-6]. The muscle originates at the medial epicondyle of the humerus and the antebrachial fascia, with the muscle belly partially fused with the adjacent muscles [3, 4]. The muscle belly turns into the long tendon and inserts in the palmar aponeurosis [2-4]. The PL muscle is located between the

\footnotetext{
* Correspondence: lukasz.olewnik@umed.lodz.pl; https://twitter.com/Oli0303 'Department of Normal and Clinical Anatomy, Interfaculty Chair of Anatomy and Histology, Medical University of Lodz, ul. Narutowicza 60, 90-136 Łódź, Poland

Full list of author information is available at the end of the article
}

flexor carpi radialis and the pronator teres muscles $[3,5]$. Its course partly overlaps the median nerve $[2,3]$.

The palmaris longus muscle, just like the plantaris muscle, presents morphological variability $[7,8]$. Variations in PL morphology are not uncommon: they may include an additional belly of the muscle, fusion with another muscle, bifurcated tendon, reversed muscle, an atypical tendon course and inserts, or multiple tendinous insertion [2, 6, 9-12]. A knowledge of PL variations is important due to its role in vascular-neural compression $[3$, $13,14]$, and its use as a graft for tendon/ligament reconstruction $[2,5,6,9,11,12,15,16]$; especially in reconstructive hand surgery $[2,5,6,9,11,12,15-17]$. It is most 
frequently used as a graft for the finger flexors $[15,17]$. The PL muscle tendon is a perfect choice for grafts, because it meets the most important criteria, such as the necessary length and diameter, and the functionality of the upper limb is undisturbed by the harvesting of the PL tendon. Furthermore, as the myofascial relationships between the muscles, tendons and adjacent structures may play an important role in understanding both functional nuances and background of specific clinical symptoms [18], the myofascial relationships of PL may play a role in the pathogenesis of the carpal tunnel syndrome [18]. The previous classifications were anatomical; however, as the PL tendon is so suitable for transplantation, due to its length, an accurate classification should be introduced which will take into account the tendon-to-belly ratio and great morphological variability. The strength of our proposed classification is that is has these two features and also describes the relationship between the palmaris tendon and median nerve.

The purpose of this study is to suggest a new classification of the palmaris longus based on its morphological variability, and to determine the relationship of the muscle to the median nerve as well as antebrachial fascia, flexor retinaculum and palmar aponeurosis. In our opinion, the new classification will be of great value in planning the transplantation of this tendon.

\section{Methods}

Eighty randomly-selected isolated (40 right and 40 left) upper limbs were obtained from adult cadavers, and fixed in $10 \%$ formalin solution before examination.

The Local Bioethics Commission issued a consent for the study (agreement no. RNN/92/16/KE).

A dissection of the forearm and hand area was performed by traditional techniques $[7,8,19,20]$. Upon dissection, the morphology of the palmaris longus was assessed, together with the location and type of its insertion to the palmar aponeurosis.

The next stage comprised a set of morphometric and anthropometric measurements of the belly and tendon of the palmaris longus muscle. The distance from the midpoint of the interstyloid line (a line drawn between the styloid processes of the radius and the ulna) and the crossing point the median nerve and the PL muscle tendon were assessed. An electronic digital calliper was used for all measurements (Mitutoyo Corporation, Kawasaki-shi, Kanagawa, Japan). Each measurement was carried out twice with an accuracy of up to $0.1 \mathrm{~mm}$. All morphometric measurements were subjected to statistical analysis.

The tendon-to-muscle ratio, defined as the relationship between the length of the tendon and the length of the muscle belly, was calculated. Based on this coefficient, types I-II were subdivided into subtypes A (tendon-to muscle-ratio $<1$ ), B (tendon-to muscle-ratio $1-1.5$ ) or $\mathrm{C}$ (tendon-to muscle-ratio $>1.5$ ).

The statistical analysis was performed using Statistica 12 software (StatSoft Polska, Cracow, Poland). A $p$-value below 0.05 was considered significant. The results are presented as a mean and standard deviation unless otherwise stated.

The normality of the continuous data distribution was checked with the Shapiro-Wilk test. As the data was not normally distributed, the Mann-Whitney test was used to compare the anthropometric measurements between two types of the palmaris longus muscle. The correlation of continuous variables was assessed with the Spearman's rank correlation coefficient.

\section{Results}

Three types of palmaris longus muscle were recognised based on variations in its insertion (Types I, II, III) Table 1. Type I and Type II were further divided into subtypes $A, B$ and $C$ based on variations in the tendonto-muscle length ratio (Table 2). All types originated on the medial epicondyle of the humerus.

Type I was characterised by the origin of the muscular part of the medial epicondyle of the humerus, with the muscle belly turned into the tendon and the insertion located on the palmar aponeurosis. This type was observed in 63 upper limbs (78.8\%) [Figs. 1a, 2a].

Type II demonstrated a proximal attachment with the same morphology as Type I. However, the tendon was bifurcated: the lateral division of the tendon always predominated and was inserted in the palmar aponeurosis, the mean length of the tendon being $39.7 \mathrm{~mm}$, while the medial division of the tendon was auxiliary and inserted in the flexor retinaculum of the wrist (mean length $26.5 \mathrm{~mm}$ ). The mean distance from the interstyloid line, between the styloid processes, and the tendon bifurcation point was $32.9 \mathrm{~mm}$. This type was found in 10 upper limbs (12.5\%) [Figs. 1b, 2b].

Type III was categorised as 'rare variations'. Namely, in one limb, the palmaris longus muscle was observed to be fused with the flexor carpi ulnaris muscle. In the distal part of the tendon, the insertion is visible in the palmar aponeurosis and in the pisiform bone. This type was found in only $1.2 \%$ of cases [Figs. 1c, 2c].

The Type I tendon predominated throughout the whole group (63 cases, 78.8\%). Type II was found in 10 cases $(12.5 \%)$. The coefficient lengths of the palmaris tendon and the belly ranged from 0.50 to 2.32 (mean $1.4 \pm 0.36$ ). The distribution of the types and subtypes of the palmaris longus tendon is presented in Table 2 .

In the Type I group, low bipennation of the muscle was found in five cases (6.25\%) [Fig. 3], while the tendon was located adjacent to the muscle belly in the other 75 cases (93.75\%). In all cases, the muscle belly was 
Table 1 Classification of Types and Subtypes of the palmaris longus muscle

\begin{tabular}{llll}
\hline Type & Inclusion criteria for the type & Subtype & Inclusion criteria for the subtype \\
\hline Type I & $\begin{array}{l}\text { • origin of the muscular part of the medial epicondyle of the humerus } \\
\text { - the tendon was inserted to the palmar aponeurosis }\end{array}$ & A & Tendon to muscle ratio $<1$ \\
& & B & Tendon to muscle ratio $1-1,5$ \\
Type II & $\begin{array}{l}\text { - origin of the muscular part of the medial epicondyle of the humerus } \\
\cdot \text { the tendon was bifurcated (lateral and medial division); the lateral } \\
\text { division was inserted to the palmar aponeurosis, and medial division } \\
\text { was inserted to the flexor retinaculuum of the wrist }\end{array}$ & A & Tendon to muscle ratio $>1,5$ \\
& "Rare variants" non-conforming definition Types I and II & B & Tendon to muscle ratio $<1$ \\
Type III & & & Tendon to muscle ratio $1-1,5$ \\
\hline
\end{tabular}

located deep to the antebrachial fascia at the site where the bicipital aponeurosis blends in the antebrachial fascia.

The dimensions of the palmaris longus tendon and the median nerve at the point of their crossing are presented in Table 3. The nerve was significantly thicker and wider than the tendon. However in $15 \%$ of cases, the tendon was wider than the nerve, and in $8 \%$, the structure was thicker. Moreover, there was a significant positive correlation between both thickness and width of the tendon and the nerve $\left(R^{2}=0.43, p=\right.$ 0.029 and $\mathrm{R}^{2}=0.41, p=0.038$ respectively).

The mean distance from the midpoint of the interstyloid line to the point where the median nerve crosses the palmaris longus tendon was $31.6(\mathrm{SD}=7.2) \mathrm{mm}$. The typical relationship between the course of the palmaris longus muscle and the median nerve is presented in Fig. 4. There was no significant difference between tendon types with regard the mean distance from the interstyloid line to the crossing of the tendon and the median nerve $(p=0.4480)$. However, in Type II, the tendon split in the region where it crossed the median nerve. The mean distance from the interstyloid line to the crossing and to the split were $31.6(\mathrm{SD}=7.2)$ and $32.9 \mathrm{~mm}(\mathrm{SD}=$ $1.4)$, respectively $(p=0.5621)$.

The comparison of morphometric measurements between two types of the palmaris longus muscle tendon shows that only the width of the muscle tendon in its distal portion was significantly lower in Type II than Type I (Table 4). The palmaris longus muscle was found to be absent in six limbs: two left and four right ones (7.5\% cases).

Table 2 Distribution of types and subtypes of the palmaris longus tendon

\begin{tabular}{llll}
\hline $\begin{array}{l}\text { Palmaris } \\
\text { longus } \\
\begin{array}{l}\text { tendon } \\
\text { types }\end{array}\end{array}$ & \multicolumn{2}{l}{ Palmaris longus tendon subtype [n (\%)] } \\
\cline { 2 - 4 } I $(n=63)$ & $12(19.1)$ & $27(42.9)$ & $\mathrm{C}$ \\
$\|(n=10)$ & $3(30.0)$ & $3(30.0)$ & $24(38.1)$ \\
\hline
\end{tabular}

\section{Discussion}

The palmaris longus is one of the most variable muscles in the human body [3, 4], and one which demonstrates morphological variability in both the muscle belly and its tendon. The descriptions found in the literature are mainly focused on the morphology of the muscle $[3,4,9,10,12-17,21,22]$. Although a classification of muscle structure types based on the ratio between the length of the belly and that of the tendon may certainly be useful in procedures based on palmaris tendon grafts, no such classification currently exists.

Possibly the first description of the anatomical variations of the PL muscle was given by Anson et al. [19]. Their study on 1600 limbs found the PL to be absent in 205. The incidence of anomalies of all types, eclusive of agnesis, was 46 in 530 consecutive arms. One half of these anomalies (23 in 46) comprised variations in position and form, while the remainder comprised accessory slips and substitute structures (15 examples), duplication of the PL (four times) and aberrancies of attachment (three times) [19].

In a study performed on 48 upper limbs, Mathew et al. [3] classify samples as normal morphology, complete agenesis and other morphological types [3]. The most common type was 'normal palmaris longus', characterised by an origin located on the medial epicondyle and an insertion to the palmar aponeurosis: This type was found in 39 upper limbs (81.25\%) [3]. The corresponding type in the present study (Type I) was found in 63 limbs (78.8\%).

Among other varieties of palmaris longus, Mathew et al. [3] identify reverse palmaris longus, characterised by a tendinous origin located on the epicondyle of the humerus, with the origin of the muscle belly on the lower $2 / 3$ of the forearm and inserted to the flexor retinaculum and the pisiform bone [3]; this type was only identified in one limb (2.08\%). They also report the occurrence of an accessory PL in one limb (2.08\%) and a 'fleshy' type in another (2.08\%), the latter being characterised by apprearing broad, fleshy and bipennate in its upper half but unipennate in the distal third, with fibres 

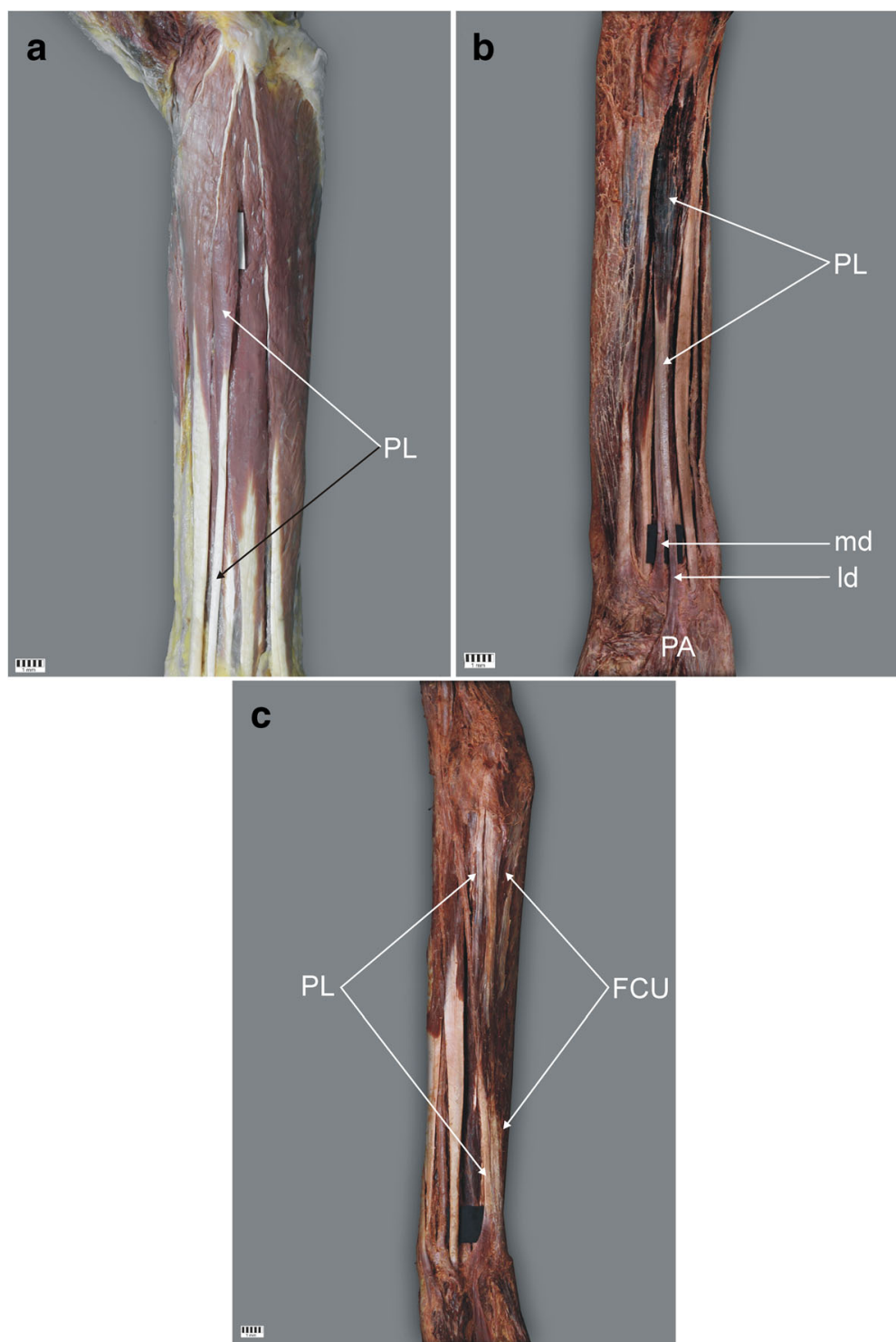

Fig. 1 Palmaris longus muscle. a Type I of the palmaris longus muscle. Right forearm. b Type II of the palmaris longus muscle. Left forearm. c Type III of the palmaris longus muscle. Right forearm. PL palmaris longus muscle, PA palmar aponeurosis, FCU flexor carpi radialis muscle, md medial division of the tendon of palmaris longus muscle, Id lateral division of the tendon of palmaris longus muscle

fanning out from the radial side all the way to its insertion. They also note a fourth or fusifom type of palmaris longus, characterised by a belly occupying the central one-third, which is originated and inserted by means of thin tendons in the upper and lower thirds, respectively. This type deprives the subject of a source of a lengthy tendon (1 case $-2.08 \%)$ [3].

None of the above types were found in the present study. However, their final 'rare variation' is similar to our Type II, labelled the 'bifurcated tendon'; it is characterised by a bifurcation of the tendon into medial and lateral parts with the medial part inserted in the palmar aponeurosis and the lateral part in the flexor retinaculum of the wrist (one case $-2.08 \%$ ) [3]. This type was identified in 10 limbs in the present study as Type II (12.5\%).

Some variants of the palmaris longus muscle have also been described as case reports. Bernardes et al. [12] describe a combined variation of palmaris longus and flexor digitorum superficialis: in this case, the palmaris longus tendon was found to pass beneath the flexor retinaculum inserted at the base of the middle phalanx of the fourth digit and replace the tendon of the flexor digitorum superficilis [12]. Marpalli et al. [6] and Murabit et al. [9] have reported the presence of the reverse palmaris longus muscle as case studies. Igbal et al. [11] 

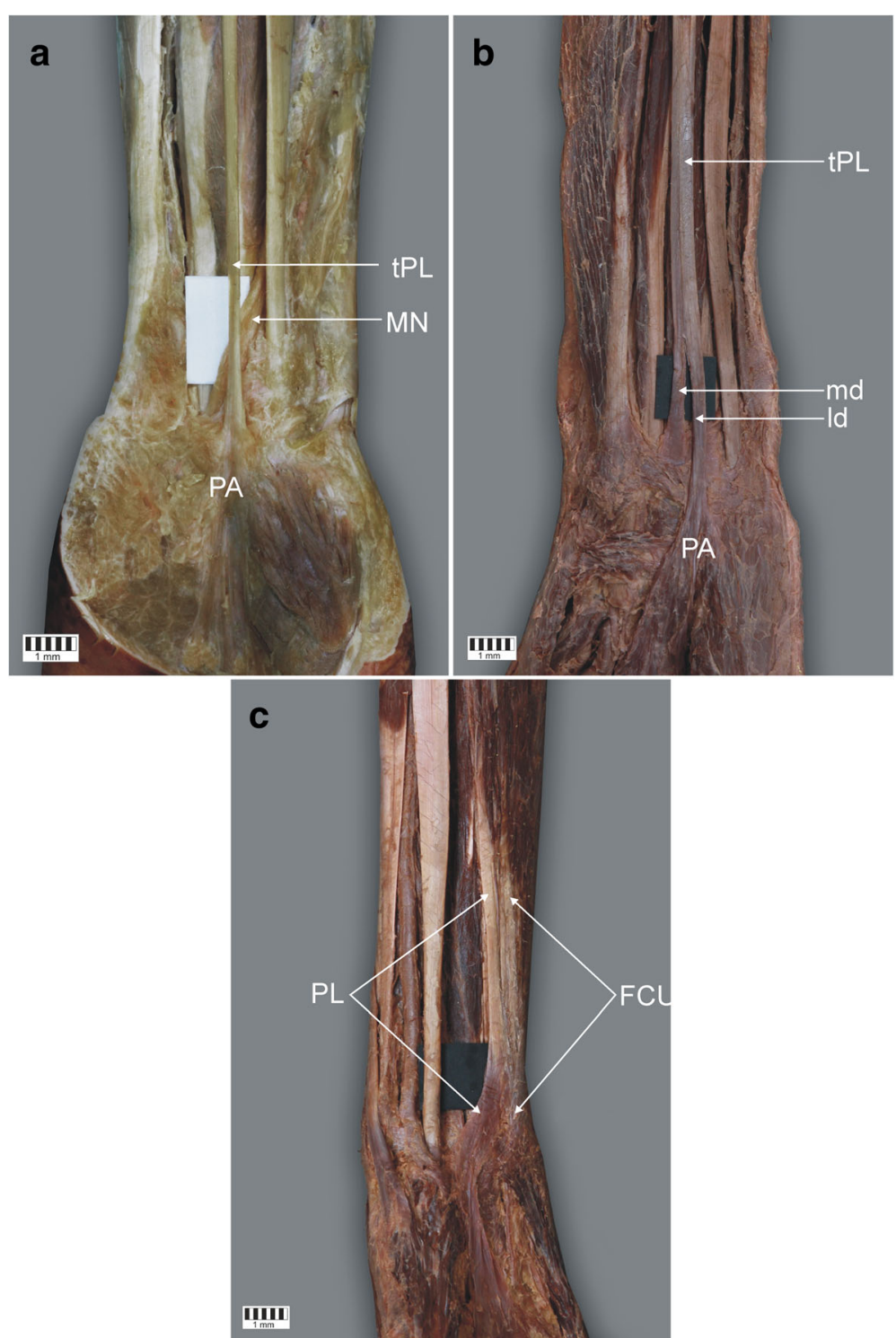

Fig. 2 Insertion of the tendon of palmaris longus muscle. a Insertion of Type I of the palmaris longus muscle. Left forearm. $\mathbf{b}$ insertion of type II of the palmaris longus muscle. Left forearm. $\mathbf{c}$ Insertion of type III of the palmaris longus muscle. Right forearm. tPL tendon palmaris longus muscle, MN Median nerve, PA palmar aponeurosis, md medial division of the tendon of palmaris longus muscle, Id lateral division of the tendon of palmaris longus muscle, FCU Flexor carpii radialis

describe the occurrence of a bitendinous PL muscle, while Barkats [10] describe a case of hypertrophy of the palmaris longus muscle as a rare anatomical variation. Kumar et al. [2] identify a rare variant of the PL with multiple tendinous insertions.

In our opinion, the case reports given above, including those described by Mathew et al. [3] as other morphological types (except the 'bifurcated tendon' type) should be classified as rare variants of the PL muscle and included into the Type III classification proposed in this paper. Every other morphological type described as a case report or differing from Types I and II might also be included in this group.
The PL was found to be absent in six limbs examined in the present study (7.5\% cases). Similarly, Mathew [3] report the absence of a PL in four limbs (8.35\%). Numerous other papers refer to the frequency of occurrence of this muscle in specific populations. Namely, Ceyhan et al. [21] found this muscle to be absent in $63.9 \%$ of a studied Turkish population, and another study reports a very high absence in an Egyptian population (50.8\%) [23]. In contrast, the muscle was found to be absent in only $1.5 \%$ of limbs in a Zimbabwean population [24], and $3.1 \%-3.8 \%$ in a Ghanaian population [25]. Sebastin et al. [26] describe a lack of the palmaris longus muscle in $4.6 \%$ limbs in an Asian population. 


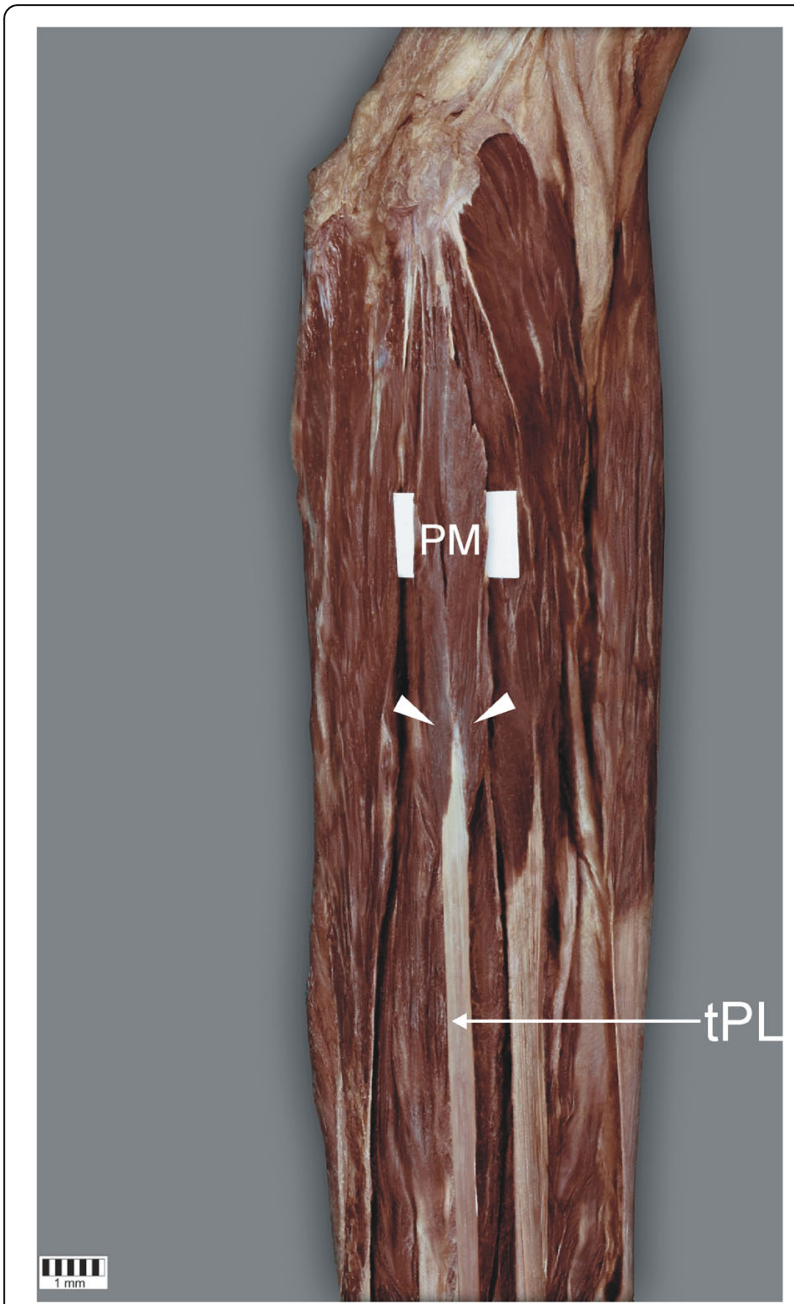

Fig. 3 Muscle with low pinnate. The white arrowheads indicate the pennation. PM palmaris longus muscle, tPL tendon palmaris longus muscle

Attention should be drawn to the specific fascial relationships between the PL and the antebrachial fascia as well as the palmar aponeurosis. Typically, the muscle is situated deep inside the antebrachial fascia. Its tendon, however, moves to a suprafascial plane in the lower third of the forearm and is in continuity with the longitudinal fibers of the palmar aponeurosis [18]. According to Stecco et al. [18] the PL should be considered as a tensor of the superficial fascial system of the subcutaneous

Table 3 Dimensions of the median nerve and tendon of palmaris longus muscle where they cross

\begin{tabular}{lllll}
\hline Dimension of structure & Mean $[\mathrm{mm}]$ & $\mathrm{SD}[\mathrm{mm}]$ & p-value \\
\hline Thickness & Tendon & 1.14 & 0.43 & 0.0056 \\
\multirow{2}{*}{ Width } & Nerve & 1.94 & 0.55 & \\
& Tendon & 3.82 & 0.96 & 0.0000 \\
& Nerve & 4.54 & 1.23 & \\
\hline
\end{tabular}

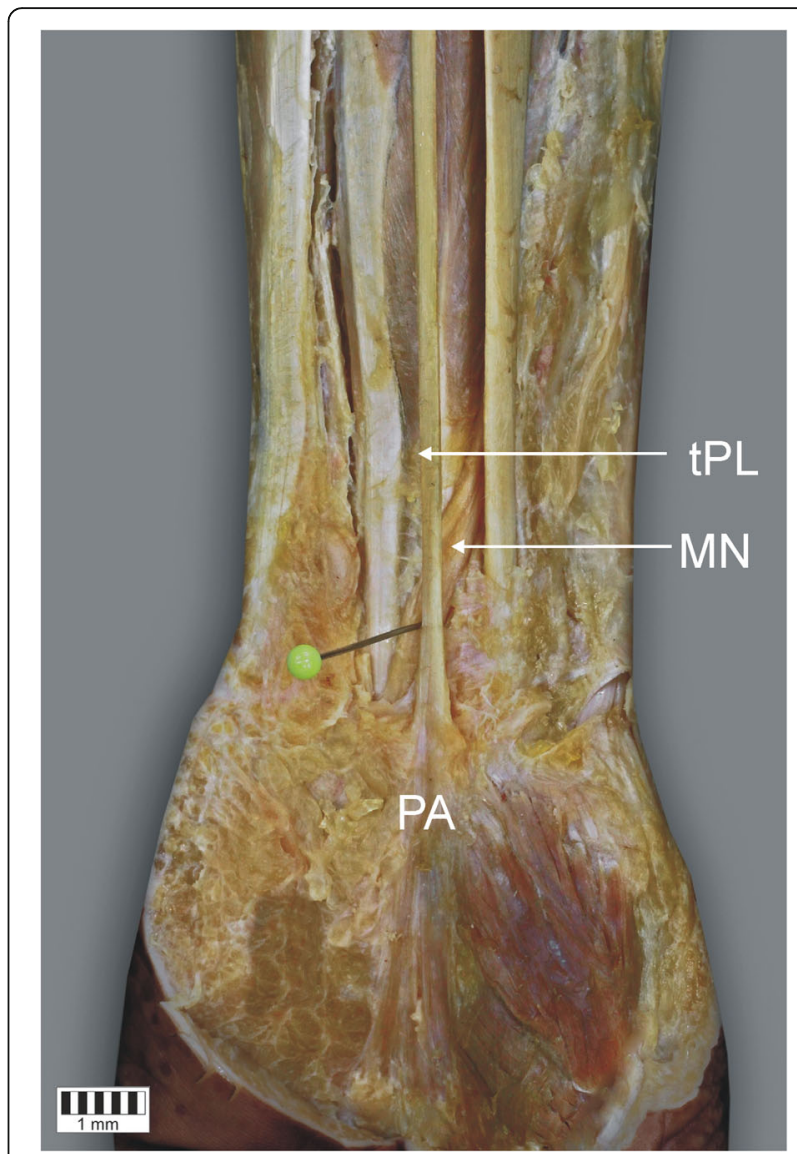

Fig. 4 Relationship between median nerve $(M N)$ and the tendon of the palmaris longus muscle ( $t P L)$

tissue. Occasionally, the PL tendon may shorten and become calcific and brittle with age, resulting in discomfort and even pain in the palm of the hand. According to Keese et al. [1] the PL tendon may play an important role in the pathophysiology of carpal tunnel syndrome and is a strong independent risk factor for carpal tunnel syndrome. Moreover, Keese et al. [1] report that "the prevalence of palmaris longus agenesis was significantly lower in the carpal tunnel group". The counterpart of the PL in the leg is the plantaris muscle, and the plantaris muscle may also play an important role in Achilles, plantaris, or calf pain syndromes [7, 8], Smith et al. [27] emphasize the need for a sonographic classification system for plantaris tendon anatomy and motion with the goal of differentiating normal from pathologic states, and identifying risk factors for symptom development. Similar recommendations may be applied to the anatomy and motion of the PL tendon - especially with regard to age and eventual presence of the clinical symptoms.

Due to the fact that the PL muscle tendon is primarily used for grafts, Cetin et al. [22] found that people with the absence of the muscle reported no ailments related 
Table 4 Comparison of measurements between types I and II of the palmaris longus muscle

\begin{tabular}{|c|c|c|c|}
\hline \multirow[t]{2}{*}{ Morphometric parameter } & \multicolumn{2}{|c|}{ Type of the palmaris longus } & \multirow{2}{*}{$\begin{array}{l}p- \\
\text { value }\end{array}$} \\
\hline & Type I & Type II & \\
\hline Forearm length [mm] & $254.8(24.3)$ & $252.0(13.6)$ & 0.7181 \\
\hline Forearm circumference [mm] & $212.5(24.8)$ & $217.2(22.0)$ & 0.5107 \\
\hline Width between processes & $55.4(4.2)$ & $54.1(4.0)$ & 0.2788 \\
\hline Thickness between processes & $163.9(21.4)$ & $170.2(15.5)$ & 0.1883 \\
\hline The length of the belly & $115.0(21.6)$ & $107.4(19.5)$ & 0.1510 \\
\hline The length of the tendon & $155.9(29.4)$ & $140.0(32.7)$ & 0.2614 \\
\hline The thickness of the tendon at the 'muscle-tendon' junction & $1.7(0.7)$ & $2.2(0.7)$ & 0.0831 \\
\hline The width of the tendon at the 'muscle-tendon' junction & $3.7(1.6)$ & $4.0(1.7)$ & 0.9361 \\
\hline The thickness of the tendon at the insertion point & $2.1(1.0)$ & $1.6(0.4)$ & 0.0905 \\
\hline The width of the tendon at the insertion point & $4.4(1.4)$ & $3.0(1.0)$ & 0.0018 \\
\hline The width of the central portion of the tendon & $4.0(1.2)$ & $3.4(1.2)$ & 0.1216 \\
\hline The width of the tendon where the tendon crosses the median nerve & $3.9(0.9)$ & $3.4(1.7)$ & 0.4222 \\
\hline The thickness of the tendon where the tendon crosses the median nerve & $1.2(0.4)$ & $1.1(0.2)$ & 0.7788 \\
\hline $\begin{array}{l}\text { Distance from the midportion of the interstyloid line to the point where } \\
\text { the median nerve crosses the tendon }\end{array}$ & $32.0(7.4)$ & $28.5(5.8)$ & 0.3773 \\
\hline
\end{tabular}

to their daily activities. Therefore, the graft of this tendon should presumably have no effect on the functionality of the hand [17]. Our proposed division of types I and II into subtypes will be of value while making decisions regarding the grafting of this tendon. The tendon tended to be longer in Type I muscles, which suggests that this type may be more appropriate for tendon transfer. This hypothesis is supported by the fact that the Type II tendon is bifurcated: when harvesting there is a risk that only one strip will be grasped and the tendon will be torn. Moreover the place where the tear may occur (region of tendon splitting) overlies the location where it crosses the median nerve. Therefore, an increased risk of nerve injury with the harvesting loop is associated with tearing the tendon. Proper tendon ablation is crucial in surgical procedures. The tendon ablation starts with locating the tendon at the proximal wrist crease and making the incision; incisions are then made every $5 \mathrm{~cm}$. From these short cuts, the distal tendon is removed and is pulled under the skin in the proximal direction [28]. In addition, the PL tendon is an important landmark for palm access for treating carpal tunnel syndrome, distal radial fracture and Guyon channel syndrome $[1,3,26]$.

The median nerve might be also injured due to other causes. Although no symptoms are typically associated with the anatomic variants of the palmaris longus muscle, there are some reports which suggest that the presence of the reverse palmaris muscle may cause compression on the median nerve [13, 14, 29-31]. Moreover, while our findings indicate that the median nerve was larger than the tendon at the point of their crossing in the majority of cases, this was not the case in $8-15 \%$ of specimens; in such cases, the size of the structure might be misleading and the median nerve may be potentially mistaken for a PL muscle tendon while harvesting [32].

Therefore, a detailed evaluation of the tendon-nerve crossing point is crucial when performing such surgical procedures as median nerve repair or tendon harvesting.

\section{Conclusion}

The proposed classification of the PL muscle presented above is a potentially significant one because the diversity of morphological variants of both the muscle belly and the insertion of PL is an important consideration when performing palmaris longus graft surgeries.

In addition, surgical procedures performed in this region require accurate knowledge of the mean distance between the interstyloid line and the crossing of the median nerve with the palmaris longus tendon.

\section{Abbreviations}

MN: Median nerve; PL: Palmaris longus

\section{Acknowledgements}

The authors wish to express their gratitude to all those who donated their bodies to medical science.

\section{Funding}

Authors have no financial or personal relationship with a third party whose interests could be positively or negatively influenced by the article's content. This research did not receive any specific grant from funding agencies in the public, commercial, or not-for-profit sectors.

Availability of data and materials

Please contact authors for data requests (Ph.D Łukasz Olewnik - email address: lukasz.olewnik@umed.lodz.pl). 


\section{Authors' contributions}

$Ł O$ project development, data collection and management, data analysis and manuscript writing. GW data analysis, manuscript editing. MP data collection, data analysis, manuscript editing. MP data collection, data analysis, manuscript editing. HJ data analysis, manuscript editing. MT data collection, manuscript editing. All authors read and approved the final manuscript.

\section{Ethics approval and consent to participate}

The protocol of the study was accepted by Bioethics Committee of Medical University of Lodz (resolution RNN/92/16/KE). The cadavers belong to the Department of Normal and Clinical Anatomy of the Medical University of Lodz. Each of the persons who died prematurely signed a notarial consent for the use of corpses for scientific and research purposes.

\section{Consent for publication}

Not applicable

\section{Competing interests}

The authors declare that they have no competing interests.

\section{Publisher's Note}

Springer Nature remains neutral with regard to jurisdictional claims in published maps and institutional affiliations.

\section{Author details}

Department of Normal and Clinical Anatomy, Interfaculty Chair of Anatomy and Histology, Medical University of Lodz, ul. Narutowicza 60, 90-136 Łódź, Poland. ${ }^{2}$ Polish Mother's Memorial Hospital Research Institute, Lodz, Poland. ${ }^{3}$ Department of Angiology, Interfaculty Chair of Anatomy and Histology, Medical University of Lodz, Lodz, Poland. ${ }^{4}$ Department of Trauma and Orthopaedic Surgery, Hospital of Ministry of Interior and Administration in Lodz, ul. Północna 42, 91-425 Łódź, Poland.

\section{Received: 2 June 2017 Accepted: 8 December 2017}

Published online: 19 December 2017

\section{References}

1. Keese GR, Wongworawat MD, Frykman G. The clinical significance of the palmaris longus tendon in the pathophysiology of carpal tunnel syndrome. J Hand Surg. 2006;31:657-60. Available from: http://jhs.sagepub.com/cgi/ doi/10.1016/J.JHSB.2006.07.015

2. Kumar N, Patil J, Swamy RS, Shetty SD, Abhinitha P, Rao MK, et al. Presence of multiple terndinous insertions of palmaris longus: a unique variation of a retrogressive muscle. Ethiop J Health Sci. 2014;24:175-8. Available from: http:// www.ncbi.nlm.nih.gov/pubmed/24795520

3. Mathew AJ. Versatile but Temperamental: A Morphological Study of Palmaris Longus in the Cadaver. J Clin Diagn Res. 2015; Available from: http://www.jcdr. net/article_fulltext.asp?issn=0973-709x\&year $=2015 \&$ volume $=9 \& i s s u e=2 \& p a g e=$ AC01\&issn=0973-709x\&id=5542

4. Bergman RA, Afifi AK, Miyauchi R. Anatomy Atlases: Illustrated Encyclopedia of Human Anatomic Variation. In: Anatomical Variation, Radiology Anatomy, Anatomy Atlas; 2015. Available from: http://www.anatomyatlases.org/ AnatomicVariants/AnatomyHP.shtml.

5. loannis D, Anastasios K, Konstantinos N, Lazaros K, Georgios N. Palmaris Longus Muscle's Prevalence in Different Nations and Interesting Anatomical Variations: Review of the Literature. J Clin Med Res. 2015;7:825-30. Available from: http://www.ncbi.n/m.nih.gov/pubmed/26491493

6. Marpalli S, ALS B, MRK G. A case of reverse palmaris longus muscle- an additional muscle in the anterior compartment of the forearm. J Clin Diagn Res. 2016;10:AD03-4. Available from: http://jcdr.net/article_fulltext. asp?issn $=0973-709 \times \&$ year $=2016 \&$ volume $=10 \&$ issue $=3 \&$ page $=$ AD03\&issn $=$ 0973-709x\&id=7369

7. Olewnik $\measuredangle$, Wysiadecki G, Polguj M, Topol M. The report on the cooccurrence of two different rare anatomic variations of the plantaris muscle tendon on both sides of an individual. Folia Morphol (Warsz). 2016; Available from: https://journals.viamedica.pl/folia_morphologica/ article/view/47711

8. Olewnik $Ł$, Wysiadecki G, Polguj M, Topol M. Anatomic study suggests that the morphology of the plantaris tendon may be related to Achilles tendonitis. Surg Radiol Anat. 2017:39:69-75. Available from: https://link springer.com/article/10.1007/s00276-016-1682-1.
9. Murabit A, Gnarra M, Mohamed A. Reversed palmaris longus muscle: Anatomical variant - case report and literature review. Can J Plast Surg. 2013; 21:55-6. Available from: http://www.ncbi.nlm.nih.gov/pubmed/24431941

10. Barkáts N. Hypertrophy of palmaris longus muscle, a rare anatomic aberration. Folia Morphol (Warsz). 2015;74:-262, 4. Available from: http:// czasopisma.viamedica.pl/fm/article/view/38905

11. Iqbal S, lqbal R, lqbal F. A Bitendinous Palmaris Longus: Aberrant Insertions and Its Clinical Impact - A Case Report. J Clin Diagn Res. 2015;9:AD03-5. Available from: http://jcdr.net/article_fulltext.asp?issn=0973-709x\&year= 2015\&volume $=9 \&$ issue $=5 \&$ page $=$ AD03\&issn $=0973-709 \times \& i d=5954$

12. Bernardes A, Melo C, Pinheiro S. A combined variation of Palmaris longus and Flexor digitorum superficialis: Case report and review of literature. Morphologie. 2016;100:245-9. Available from: http://www.ncbi.nlm.nih.gov/ pubmed/27265750

13. Yildiz M, Sener M, Aynaci O. Three-headed reversed palmaris longus muscle: a case report and review of the literature. Surgical and radiologic anatomy. Surg Radiol Anat. 2000;22:217-9. Available from: http://www.ncbi.nlm.nih. gov/pubmed/11143317

14. Bencteux P, Simonet J, el Ayoubi L, Renard M, Attignon I, Dacher JN, et al. Symptomatic palmaris longus muscle variation with MRI and surgical correlation: report of a single case. Surg Radiol Anat. 23:273-5. Available from: http://www.ncbi.nlm.nih.gov/pubmed/11e694974

15. Sharma DK, Shukla CK, Sharma V. Clinical assessment of absence of palmaris longus muscle and its association with gender, body sides, Handedness and other neighboring anomalies in a population of Central India. J Anat Soc India. 2012:61:13-20

16. Aversi-Ferreira RAGMF, Bretas RV, Maior RS, Davaasuren M, Paraguassú-Chaves $\mathrm{CA}$, Nishijo $\mathrm{H}$, et al. Morphometric and statistical analysis of the palmaris longus muscle in human and non-human primates. Biomed Res Int. 2014 2014:1-6. Available from: http://www.ncbi.nlm.nih.gov/pubmed/24860810

17. Sunil V, Rajanna S, Gitanjali KJ. Variation in the insertion of the palmaris longus tendon. Singap Med J. 2015;56:e7-9. Available from: http://www. ncbi.nlm.nih.gov/pubmed/25640108

18. Stecco C, Lancerotto L, Porzionato A, Macchi V Tiengo C, Parenti A, et al. The palmaris longus muscle and its relations with the antebrachial fascia and the palmar aponeurosis. Clin Anat. 2009:22:221-9. Available from: http://doi.wiley.com/10.1002/ca.20747

19. Anson B, McVay C. Surgical anatomy. Fifth edit ed. Philadeplphia, W.B. Sauders Company; 1958.

20. Olewnik $Ł$, Podgórski M, Polguj M, Wysiadecki G, Topol M. Anatomical variations of the pronator teres muscle in a Central European population and its clinical significance. Anat Sci Int. 2017; Available from: http://link. springer.com/10.1007/s12565-017-0413-y

21. Ceyhan O, Mavt A. Distribution of agenesis of palmaris longus muscle in 12 to 18 years old age groups. Indian J Med Sci. 1997;51:156-60. Available from: http://www.ncbi.nlm.nih.gov/pubmed/9355719

22. Cetin A, Genc M, Sevil S, Coban YK. Prevalence of the palmaris longus muscle and its relationship with grip and pinch strength: a study in a Turkish pediatric population. Hand. 2013:8:215-20. Available from: http:// journals.sagepub.com/doi/10.1007/s11552-013-9509-6

23. Raouf HA, Kader GA, Jaradat A, Dharap A, Fadel R, Salem AH. Frequency of palmaris longus absence and its association with other anatomical variations in the egyptian population. Clin Anat. 2013;26:572-7. Available from: http://www.ncbi.nlm.nih.gov/pubmed/23339087

24. Gangata $H$. The clinical surface anatomy anomalies of the palmaris longus muscle in the Black African population of Zimbabwe and a proposed new testing technique. Clin Anat. 2009;22:230-5. Available from: http://doi.wiley. com/10.1002/ca.2075

25. Osonuga A, Mahama HM, Brown AA, Osonuga OA, Serbeh G, Harding AN, et al. The prevalence of palmaris longus agenesis among the Ghanaian population. Asian Pac J Trop Dis. 2012;2(Supplement 2):S887-89.

26. Sebastin SJ, AYT L. Clinical assessment of absence of the palmaris longus and its association with other anatomical anomalies- a Chinese population study. Ann Acad Med Singap. 2006:35:249-53. Available from: http://www. ncbi.n/m.nih.gov/pubmed/16710495

27. Smith لـ, Alfredson $H$, Masci L Sellon لL, Woods CD. Differential plantarisachilles tendon motion: a sonographic and cadaveric investigation. Phys Med Rehab Res. 2016; [cited 2017 Feb 6]; Available from: http://inkinghub. elsevier.com/retrieve/pii/S1934148216310607

28. Lawler E, Adams BD. Reconstruction for DRUJ instability. Hand: 2007 2:123126. Available from: http://www.ncbi.n/m.nih.gov/pubmed/18780072. 
29. Meyer FN, Pflaum BC. Median nerve compression at the wrist caused by a reversed palmaris longus muscle. J Hand Surg. 1987;12:369-71. [cited 2017 Feb 6] Available from: http://www.ncbi.nlm.nih.gov/ pubmed/3584883

30. Depuydt KH, Schuurman AH, Kon M. Reversed palmaris longus muscle causing effort-related median nerve compression. J Hand Surg. 1998;23: 117-9. Available from: http://www.ncbi.n/m.nih.gov/pubmed/9571503

31. Güler MM, Celiköz B. Anomalous palmaris longus muscle causing carpal tunnel-like syndrome. Arch Orthop Trauma Surg. 1998;117:296-7. [cited 2017 Feb 6] Available from: http://www.ncbinlm.nih.gov/pubmed/9581267

32. Weber RV, Mackinnon SE. Median nerve mistaken for palmaris longus tendon: restoration of function with sensory nerve transfers. Hand ( $N$ Y). 2007;2:1-4. [cited 2017 Feb 6] Available from: http://han.sagepub.com/ lookup/doi/10.1007/s11552-006-9011-5

Submit your next manuscript to BioMed Central and we will help you at every step:

- We accept pre-submission inquiries

- Our selector tool helps you to find the most relevant journal

- We provide round the clock customer support

- Convenient online submission

- Thorough peer review

- Inclusion in PubMed and all major indexing services

- Maximum visibility for your research

Submit your manuscript at www.biomedcentral.com/submit
Biomed Central 